\title{
Caractérisation Sédimentologique Et Minéralogique Des Formations Du Tertiaire-Quaternaire De La Région D’anyama Au Sud De La Côte d'Ivoire
}

\author{
Kra Akoua Clarisse \\ Assale Fori Yao Paul \\ Yao N'Goran Jean-Paul \\ Monde Sylvain \\ Aka Kouamé
}

Université Félix HOUPHOUËT-BOIGNY d'Abidjan /

UFR des Sciences de la Terre et des Ressources Minières /

Laboratoire de Géologie Marine et de Sédimentologie

doi: 10.19044/esj.2016.v12n3p251 URL:http://dx.doi.org/10.19044/esj.2016.v12n3p251

\begin{abstract}
Anyama is located on the onshore of the sedimentary basin of Côte d'Ivoire. It is to the east of the basin and the formations, particularly those dating back to the Quaternary may have been studied. Based on samples, collected from two drill holes (An1 and An2), each $114 \mathrm{~m}$ deep, a sedimentology and mineralogy characterization of Plio-Quaternary formations was carried out. The sedimentological study was assessed by taking account the lithology, the grain size, the transport model and the environment deposit of the sediments, the morphoscopy and the exoscopy of the quartz grain. The mineralogy study was made on clays through X-Ray Diffraction (XRD). Lithological characterization, revealed the presence of clay facies (kaolinite), sandy facies and mixed sediments, all covered with the earth bar. As for the size, it indicates coarse to medium sands with a positive asymmetry to almost positive, sands moderately classified to fairly classify. The relative mineralogy reveals the presence of silicon, aluminum and iron oxides. XRD indicates that quartz and kaolinite prevail and gypsum, rutile or anatase, illite and calcium sulfate hydrate are associated minerals.
\end{abstract}

Keywords : Sedimentology, Mineralogy, Quaternary, Anyama, Côte d'Ivoire. 


\section{Résumé}

Anyama est une localité située dans la partie terrestre (Onshore) du bassin sédimentaire de la Côte d'Ivoire. Elle est plus à l'Est du bassin et les formations, notamment, celles datant du quaternaire ont été peut étudiées. A partir d'échantillons recueillis, issus de deux forages (An1 et An2), profonds chacun de $114 \mathrm{~m}$, une caractérisation sédimentologique et minéralogique des formations du Plio-Quaternaire a été effectuée. L’étude sédimentologique a été appréciée en tenant compte de la lithologie, de la granulométrie, du mode de transport et de l'environnement de dépôt des sédiments, de la morphoscopie et de l'exoscopie des quartz, tandis que, la minéralogie s'est faite sur les argiles à travers la diffraction des rayons $\mathrm{X}$ (DRX). La caractérisation lithologique a révélé la présence de faciès argileux, constitué d’argile (bariolée et grisâtre), de faciès sableux et de sédiments mixtes, tous recouverts de la terre de barre. Quant à la granulométrie, elle indique des sables grossiers à moyens, avec une asymétrie positive à presque positive, des sables moyennement classés à assez bien classés. La DRX indique que le quartz et la kaolinite prédominent et que le gypse, le rutile ou l'anatase, l’illite et les sulfates de calcium hydratés sont les minéraux associés.

Mots-clés : Sédimentologie, Minéralogie, Quaternaire, Anyama, Côte d'Ivoire.

\section{Introduction :}

Le domaine sédimentaire de la Côte d'Ivoire recouvre 2,5\% de son territoire (Tagini, 1971 ; Yacé, 2002). Il forme une mince frange littorale, en bordure de l’océan atlantique, dans le Golfe de Guinée septentrional et, est d’âge secondaire-tertiaire (Tagini, 1972 ; Bessoles, 1977) à quaternaire (Martin et Tastet, 1972). Depuis les années 2000, des études ont été réalisées sur la partie onshore qui en donnent des orientations, mais la proportion sur le quaternaire, reste faible. En effet, qu'il s'agisse de travaux sur la biostratigraphie (N’Da et al., 1995 ; Goua, 1997 ; Digbehi et al., 1994, 2011 ; Bamba et al., 2011), sur la sédimentologie (Digbehi et al., 1993), etc., le ratio, études du tertiaire (crétacé) par rapport au quaternaire reste élevé. Cependant, quelques orientations ont été indiquées sur la transition Mio-PlioQuaternaire par Digbehi et al. (2001) dans la localité de Bingerville. Aussi Yao et al. (2011) ont permis de faire une révision des formations de Fresco, à l'Ouest du bassin, tout comme Gbangbot et al. (2012), qui, de son côté a identifié à Adjamé, Abobo, Yopougon, Assinie et Bingerville, l’origine et préciser les facteurs et les phénomènes qui interviennent dans le transport et le dépôt des sédiments dans le Sud-Est du bassin onshore. De plus, avec Assalé (2013), Assalé et al. (2013), l’on a pu comprendre la distribution des 
cortèges sédimentaires dans la partie Est du bassin, notamment à Alépé, Bonoua, Adiaké, Eboinda, Samo.

Sur la base des quelques résultats présentés, qui, d’ailleurs, sont très probants, des localités restent à étudier afin de mieux comprendre le fonctionnement de la partie onshore du bassin ivoirien. C'est dans ce cadre, que la présente étude est menée à Anyama. A partir d'une approche sédimentologique et minéralogique, des indications sur les formations du Plio-Quaternaire seront données et permettront de déduire les implications paléoenvironnementales.

\section{Localisation de la zone d'étude :}

\section{Cadre géographique}

Située à 22 km d'Abidjan, la localité d’Anyama fait partie du district d'Abidjan. Elle est localisée entre les latitudes $5^{\circ} 10$ et $5^{\circ} 35$ Nord et les longitudes $3^{\circ} 45$ et $5^{\circ} 20$ Ouest. Elle couvre une superficie d'environ $370 \mathrm{~km}^{2}$. Les limites physiques sont constituées par le contact socle cristallin - bassin sédimentaire au Nord, le Golfe de Guinée au Sud, le fleuve côtier la Mé et les lagunes Aghien et Potou à l'Est et le fleuve Agnéby à l'Ouest.

\section{Cadre géologique}

La Côte d'Ivoire est subdivisée en deux domaines géologiques dont $97,5 \%$ de socle cristallin et $2,5 \%$ de bassin sédimentaire dont fait partie la localité d’Anyama. Le bassin sédimentaire est composé de dépôts continentaux et marins dont les plus anciens sont d’âge crétacé moyen. Certains auteurs remontent jusqu'au Crétacé inférieur et même jusqu’au Jurassique supérieur (Assalé, 2013). La localité d’Anyama est située dans le bassin onshore précisément au Sud -Est de la Côte d’Ivoire.

\section{Localisation des puits}

Deux forages (An1 et An2) ont été réalisés dans ce bassin dans la localité d'Anyama. Le puits An1 est situé au quartier PK18 dans l'extension Nord de la commune d'Abobo. Le puits An2 est foré au Nord-Est du premier puits, dans le quartier Belle ville. Ces deux puits sont distants de deux kilomètres (Figure 1). Les coordonnées géographiques de ces différents puits sont présentées dans le tableau I ci-dessous.

Tableau I : Coordonnées en UTM des puits forés à Anyama

\begin{tabular}{cccc}
\hline Puits & Longitude $(\mathrm{m})$ & Latitude $(\mathrm{m})$ & Altitude $(\mathrm{m})$ \\
\hline Anyama 1 (An1) & 0383096 & 0602811 & 136 \\
\hline Anyama 2 (An2) & 0384533 & 0603989 & 135 \\
\hline
\end{tabular}




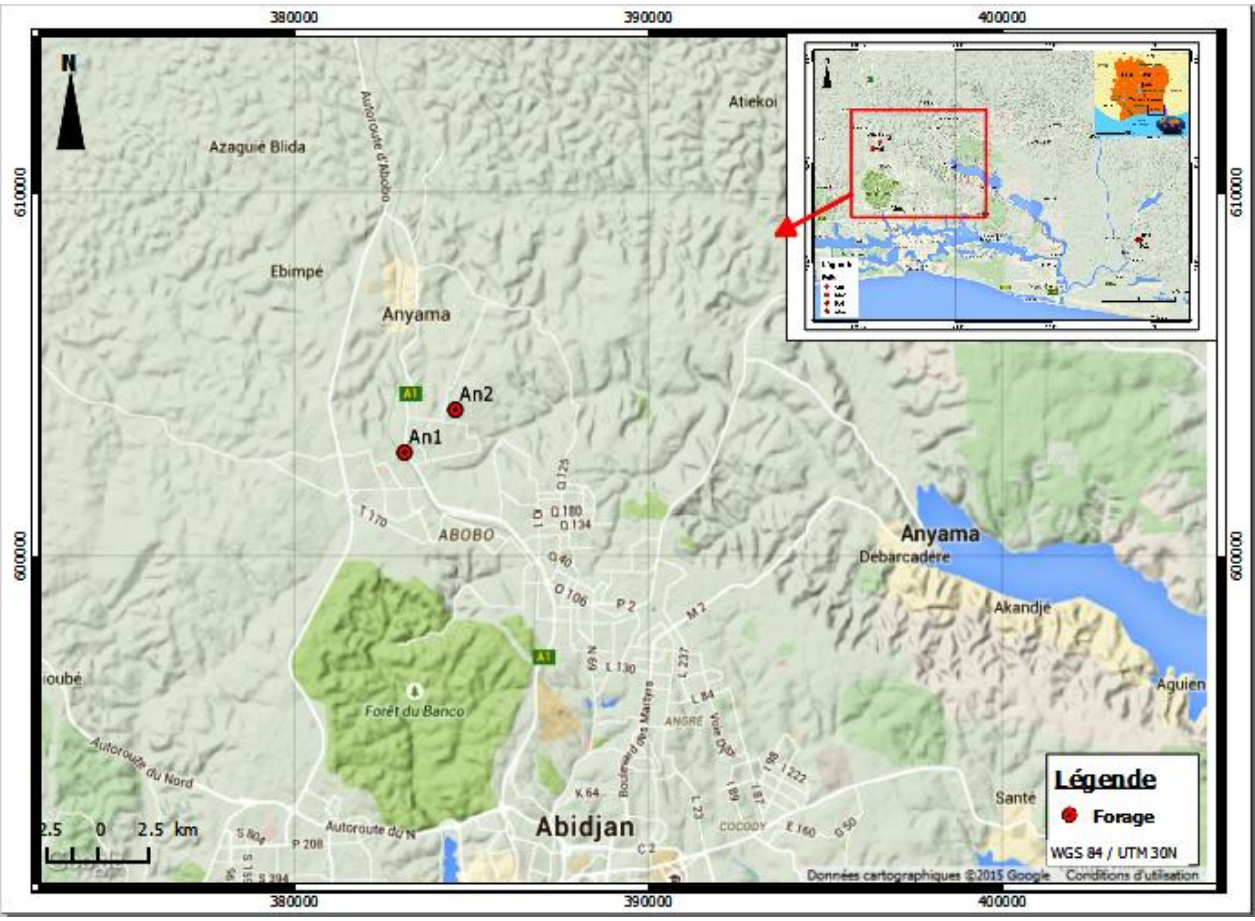

Figure 1 : Localisation des puits à Anyama

\section{Matériel et méthodes d'études}

L'approche méthodologique adoptée se résume en des études lithologique, granulométrique et minéralogique, afin de déterminer le mode et l'environnement de dépôt.

La lithologie est établie à partie de la description macroscopique des sédiments, respectivement, à l'œil nu et à la loupe binoculaire.

Quant à la granulométrie dont l'objectif est de mesurer la dimension des grains et de connaître la fréquence statistique des différentes tailles qui constituent les sédiments sableux, elle s'est faite à l'aide d'une colonne de tamis de type AFNOR. Ainsi, les sédiments sont préalablement lavés à l'eau savonneuse, plusieurs fois, pour éliminer les fractions argileuses et désolidariser les grains. Ils sont par la suite décarbonatés à l'acide chlorhydrique $(\mathrm{HCl})$ à $16 \%$. Pour les sédiments contenant la matière organique, ils sont trempés dans l'eau oxygénée en vue de leur élimination. Enfin, le sédiment propre obtenu est mis à l'étuve à $60^{\circ}$ jusqu'à ce qu'il sèche. Par la suite, $100 \mathrm{~g}$ de ce sédiment sec sont prélevés pour le tamisage, pesé puis placé au sommet d'une colonne de 16 tamis AFNOR. Ces tamis sont superposés dans l'ordre des mailles de tailles décroissantes $(500 \mu \mathrm{m}$ à $63 \mu \mathrm{m}$ ), et, au bout de $15 \mathrm{mn}$ d'agitation, le refus de chaque tamis est pesé. C'est alors que s'ensuit la phase analytique et la phase interprétative, tel qu'indiqué par Folk et Ward (1957), Tricart (1965). Cette phase analytique 
permet de connaitre la distribution des dimensions des particules du sédiment (obtention de courbes cumulatives et courbes de fréquences) à partir des paramètres granulométriques $(\mathrm{Mz}$, So, Sk). Ceux-ci se définissent comme suit :

- la moyenne $(\mathrm{Mz})$ représente la distribution granulométrique moyenne des sédiments. Elle traduit la puissance moyenne des paléoécoulements. Elle est déterminée par la formule suivante : $\mathrm{Mz}$ $=(\mathrm{Q} 16+\mathrm{Q} 50+\mathrm{Q} 84) / 3$

- l'écart-type ou classement $(\sigma)$ permet d'apprécier le classement des sables. Sa formule est : $\sigma($ en phi $)=(\Phi 84-\Phi 16) / 4+(\Phi 95-\Phi 5) /$ 6,6

- le Skewness (Sk), traduit le degré d'asymétrie de la courbe de distribution par rapport à la médiane. Cette asymétrie indique la prépondérance ou non des particules fines (valeurs positives) ou des particules grossières (valeurs négatives) par rapport à la moyenne de l'échantillon :

$\mathrm{Sk}=(\Phi 16+\Phi 84-2 \Phi 50) / 2(\Phi 84-\Phi 16)+(\Phi 5+\Phi 95-2 \Phi 50) / 2$ (Ф95-Ф5).

La phase interprétative, avec les diagrammes de Passega (1957) et Freidman (1967), a pour action la reconstitution des caractères du (ou des) écoulement(s), responsables du transport du sédiment, et, au-delà, l'identification du milieu de sédimentation.

Concernant la minéralogie, elle a été réalisée au Diffractomètre de Rayon X (DRX) pour déterminer la composition minéralogique des argiles, en vue d'apporter des arguments sur l’origine des sédiments.

\section{Résultats et discussion}

\section{Caractérisation lithologique des formations}

\section{Lithologie du puits Anyama 1 (An1)}

Ce puits est caractérisé par trois faciès qui se présentent comme suit : l'argile bariolée, le niveau de sables quartzeux, et la terre de barre (figure 2).

Située entre les côtes 114-113 m, l'argile est bariolée avec une texture ferme à massive. La matrice argileuse contient des silts de quartz translucides et subanguleux. C'est donc une argile silteuse.

Compris entre 113 et $35 \mathrm{~m}$, ce sable forme un intervalle épais de 78 m. La couleur de ces sédiments varie du rose orangé modéré au brun orangé modéré et au jaune orangé pâle. Les grains de sables sont généralement moyens. Ils deviennent fins entre 63 et $53 \mathrm{~m}$. Ce niveau contient des grains de quartz translucides à majoritairement rougeâtres. Ce sont des grains anguleux à subanguleux. On note la présence de quelques grains subarrondis avec de rares grains arrondis. De petits bancs ferrugineux sont intercalés dans ces sables. 
La Terre de barre termine la sédimentation de ce puits. Elle est comprise entre 35 et $1 \mathrm{~m}$. Ce sont des sédiments composés d'un mélange d'argile, de silts et de sables. La couleur de ces sédiments varie du jaune orangé pâle entre 35 et $23 \mathrm{~m}$ au rougeâtre entre 22-4 m. Entre 3 et $1 \mathrm{~m}$, elle devient brun modéré a brun jaunâtre. La partie sableuse est très fine. Ce sont donc des argiles silto-sableuses. Elles sont constituées de grains de quartz translucides, à majoritairement rougeâtre et jaunâtre. Les grains de quartz sont pour la plupart anguleux à sub anguleux. On y observe des débris carbonés et des morceaux de racines vers la surface qui constitue la terre arable (sol pédologique).

\section{Lithologie du puits Anyama 2 (An2)}

La lithologique rencontrée dans le puits Anyama 2, est constituée d'argile, de sables quartzeux et de sédiments mixtes (argile sableuse et sable argileuse) (Figure 3).

Dans ce puits, l'argile bariolée débute la sédimentation. Il est compris entre les cotes $114-110 \mathrm{~m}$. L'argile bariolée, de texture ferme et massive, a une coloration jaune sombre brunâtre au brun modéré.

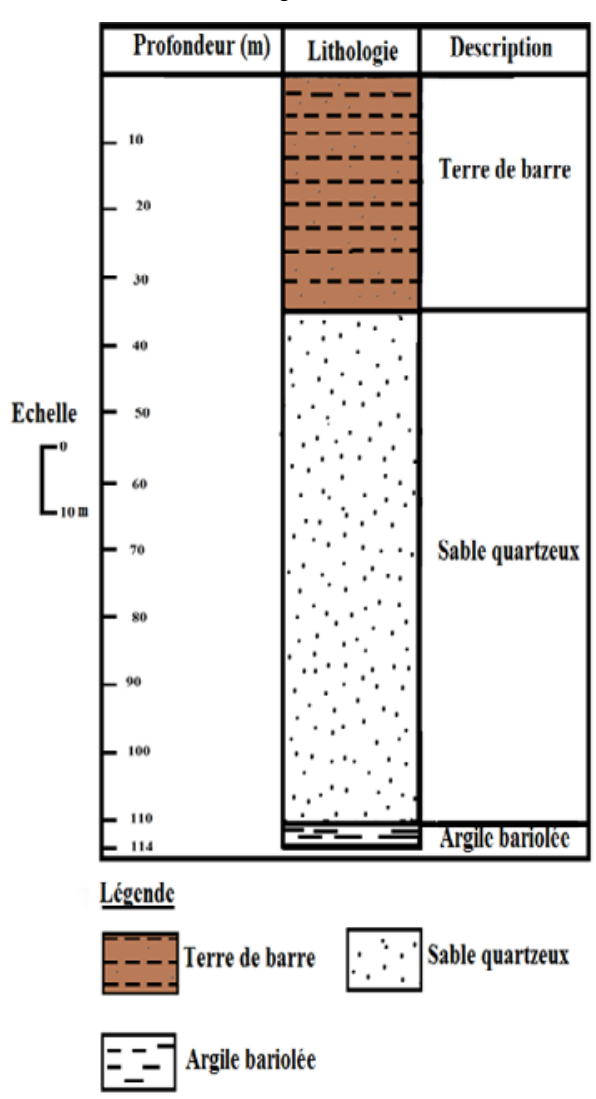

Figure 2 : $\log$ lithologique du puits An1

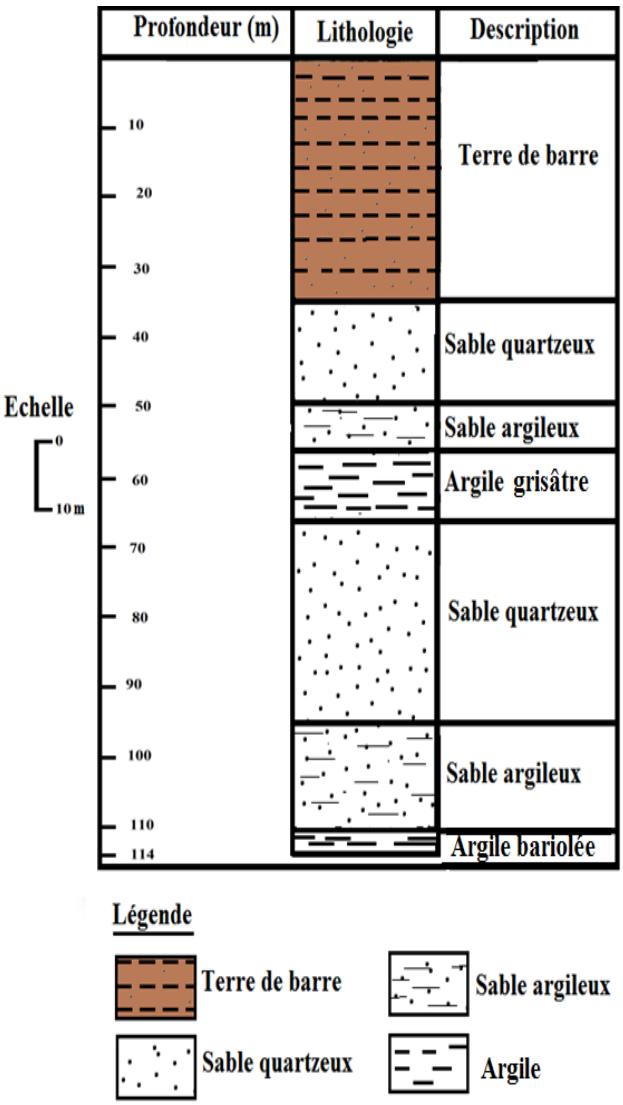

Figure 3 : $\log$ lithologique du puits An2 
La présence de la matière organique serait l'origine de la coloration noirâtre de l'argile. Cette argile est également silteuse, elle contient des silts quartzeux.

Situé entre les profondeurs 110 et $94 \mathrm{~m}$, ce sédiment a une coloration variant du brun modéré au rouge clair. Cette couche est constituée de grains de quartz moyens à grossiers entre 110 et $99 \mathrm{~m}$ et de grains de quartz fins a moyens entre 99 et $94 \mathrm{~m}$. Ce sont des quartz anguleux à subanguleux, translucides à très peu rougeâtres. La matrice argileuse est très silteuse.

Compris entre les côtes 94 et $67 \mathrm{~m}$, ce niveau est constitué de sables quatzeux à grains moyens à fins entre 94-90 m et très fins entre $84-80 \mathrm{~m}$ et 70 - $67 \mathrm{~m}$. La couleur observée varie de l'orange rougeâtre modéré au rose orangé modéré et parfois une coloration brun rougeâtre modéré. Ce sont des grains anguleux à subanguleux avec de rares grains subarrondis. Il est présent entre la côte $94 \mathrm{~m}$ et la côte $67 \mathrm{~m}$.

Une autre couche d'argile est situé entre les cotes 67 et $55 \mathrm{~m}$. C'est de l'argile très peu silteuse de couleur rose orangé grisâtre. Elle a une texture ferme.

Entre les côtes 55 et $50 \mathrm{~m}$, les sédiments sont de couleur brun rougeâtre modéré. Les grains de quartz sont subanguleux à subarrondis, translucides et rougeâtre. La fraction argileuse est rougeâtre et silteuse.

Situé entre les profondeurs 49 et $40 \mathrm{~m}$, ce niveau est constitué de sables à grains moyens à fins. La couleur de ces grains de sable varie de l'orange grisâtre au brun jaunâtre modéré et au jaune orangé pâle. Ces grains sont translucides à rougeâtres, anguleux à subanguleux.

De 39 à $30 \mathrm{~m}$ les sédiments sont très silteux et silteux de 29 à $3 \mathrm{~m}$. La couleur varie du jaune au jaune sombre orangé et à l'orange jaunâtre pâle. Pour les sédiments les plus superficiels, la coloration devient rouge brunâtre. Ils contiennent des cuirasses ferrugineuses, des débris carbonés, des morceaux de tige et des racines. Les grains de quartz sont translucides à rougeâtre, anguleux à subanguleux. La terre de barre a une épaisseur de $39 \mathrm{~m}$ qui part de la côte 39 à la surface.

\section{Etude corrélative}

Au Nord (puits Anyama 2), la sédimentation débute par le dépôt d'argile bariolée sur laquelle repose une couche de sable argileux. Ensuite vient l'épaisse couche de sable quartzeux à l'intérieur de laquelle sont intercalées les couches d'argile grisâtre et de sable argileux. Enfin la terre de barre qui coiffe l'ensemble. Cette lithologie n'est pas observée au Sud où a été foré le puits Anyama 1. Ce dernier présente en effet trois types de faciès à savoir la couche d'argile bariolée, une épaisse couche de sable quartzeux et la terre de barre (figure 4). 


\section{Caractérisation granulométrique des sédiments sableux Représentations graphiques}

Les courbes cumulatives semi-logarithmiques des sables des différents puits étudiés présentent les mêmes allures. Le faciès est de type hyperbolique ou sigmoïde (en forme de $\mathrm{S}$ ) (figure 5 ). Ce sont des courbes à pente redressées. Aussi, les courbes de fréquence des sables sont unimodales (figure 6) et, elles sont plus ou moins étalées.

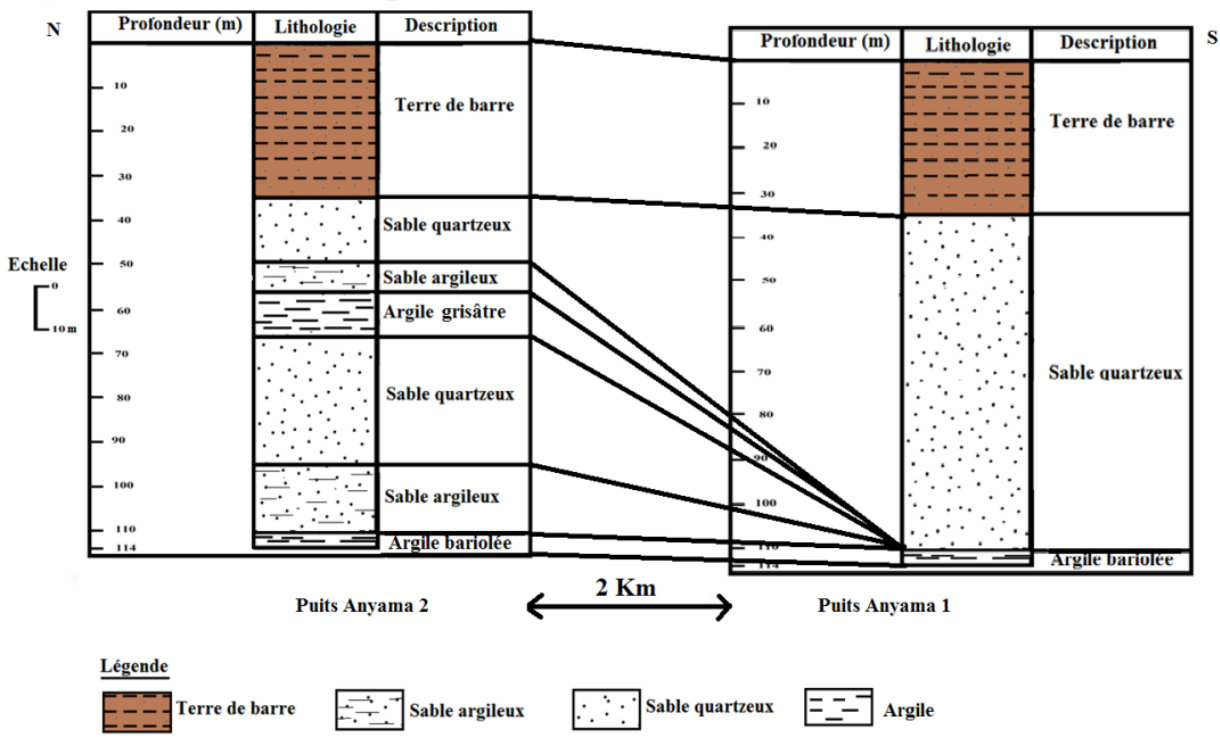

Figure 4 : Corrélation entre les puits An1 et An2

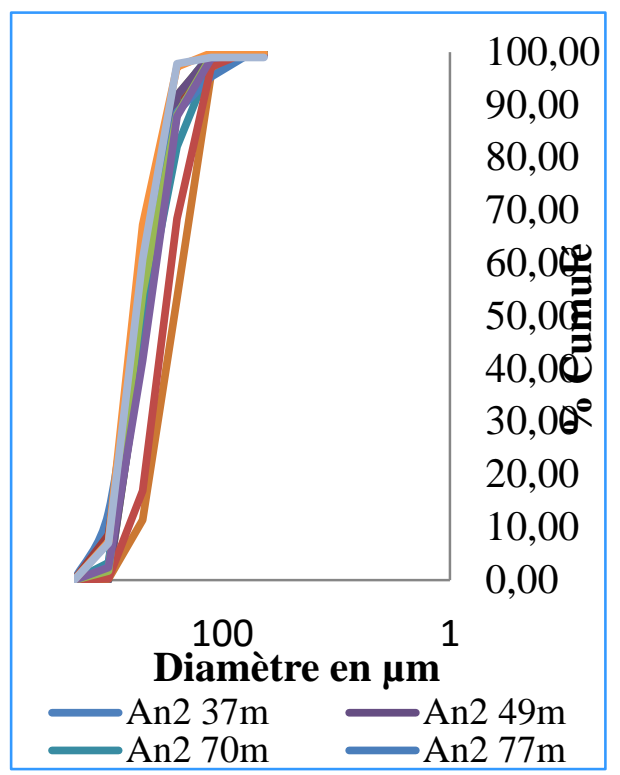

Figure 5 : Courbes cumulatives semilogarithmique de type hyperbolique

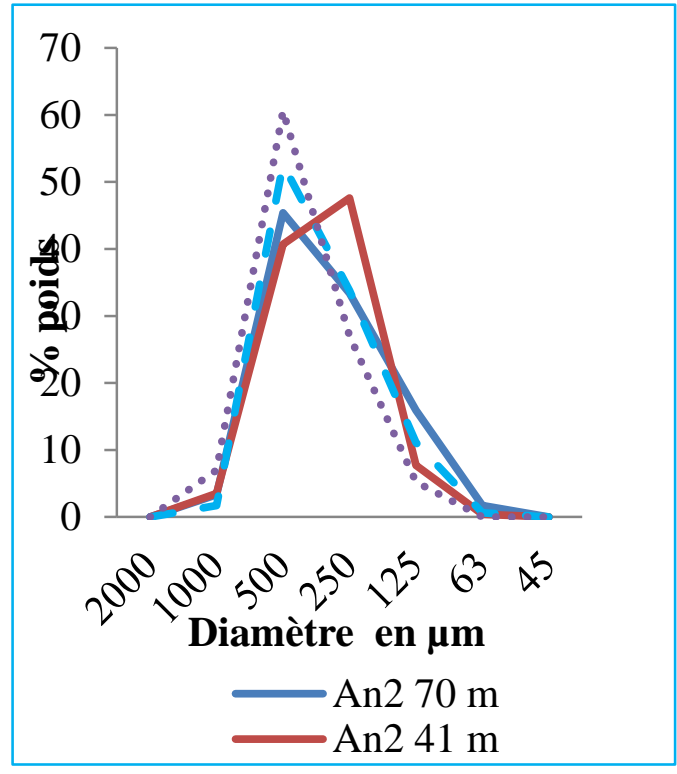

Figure 6 : Courbes de fréquence unimodales 


\section{Paramètres granulométriques des sables}

Le calcul de la moyenne, de l'écart type et du skewness ont permis de caractériser les sables de la localité d'Anyama (Tableaux II et III). Cette caractérisation révèle que les sables sont pour la plupart de taille grossière à moyenne, presque symétrique à asymétrique vers les éléments fins. En ce qui concerne l'écart-type, les sables moyennement classés prédominent avec de rares sables assez bien à bien classés.

Tableau II : Caractéristiques granulométriques des sables du puits Anyama 1

Paramètres granulométriques

\begin{tabular}{lllll} 
Echantillons & \multicolumn{3}{c}{} & \\
\cline { 2 - 4 } & $\mathbf{M z}$ & Sk & So & Description \\
\hline An1 $37 \mathrm{~m}$ & 0,498 & $-0,005$ & 0,75 & Sables moyens, presque symétriques, assez bien classés \\
An1 $45 \mathrm{~m}$ & 0,332 & 0,12 & 0,72 & Sables moyens, asymétriques vers les fins, moyennement classés \\
An1 $73 \mathrm{~m}$ & 0,517 & 0,05 & 0,77 & Sables grossiers, presque symétriques, moyennement classés \\
An1 $90 \mathrm{~m}$ & 0,662 & 0,12 & 0,76 & Sables grossiers, asymétriques vers les fins, moyennement classés \\
An1 $100 \mathrm{~m}$ & 0,607 & 0,07 & 0,79 & Sables grossiers, presque symétriques, moyennement classés \\
\hline
\end{tabular}

Tableau III : Caractéristiques granulométriques des sables du puits Anyama 2

\begin{tabular}{|c|c|c|c|c|}
\hline \multirow{2}{*}{ Echantillons } & \multicolumn{3}{|c|}{ Paramètres granulométriques } & \multirow[b]{2}{*}{ Description } \\
\hline & $\mathbf{M z}$ & Sk & So & \\
\hline An2 $37 \mathrm{~m}$ & 0,60 & 0,09 & 0,92 & sables grossiers, presque symétriques, moyennement classés \\
\hline An2 $70 \mathrm{~m}$ & 0,52 & 0,19 & 0,85 & sables grossiers, asymétriques vers les fins, moyennement classés \\
\hline An2 $77 \mathrm{~m}$ & 0,62 & 0,34 & 0,66 & sables grossiers, très asymétriques vers les fins, assez bien classés \\
\hline An2 84 m & 0,35 & 0,08 & 0,78 & sables moyens, presque symétriques, moyennement classés \\
\hline An2 $103 \mathrm{~m}$ & 0,64 & 0,16 & 0,68 & sables grossiers, asymétriques vers les fins, assez bien classés \\
\hline
\end{tabular}

\section{Mode de transport et environnement de dépôt Mode de transport des sables quartzeux}

Le diagramme de Passega (1957) a permis de déterminer le mode de transport des sables. Les points situés sur ce diagramme se retrouvent dans deux segments comme indiqué par la figure 7. Ce sont les segments PO et ON. Selon Passega (1957), chaque segment témoigne d'un mode de transport donné. Le segment PO indique que les sédiments sont transportés par roulement et suspension de fond et ON témoigne de sédiments transportés essentiellement par roulement. 
Figure 7 : Distribution des sables des puits d’Anyama sur le diagramme de Passega (1957)

\section{Environnement de dépôt des sables quartzeux}

Pour la détermination des environnements de dépôt, le diagramme de Freidman (1967) appliqué aux sables des deux puits, donne les mêmes résultats. Tous les points tombent dans le domaine de type fluviatile

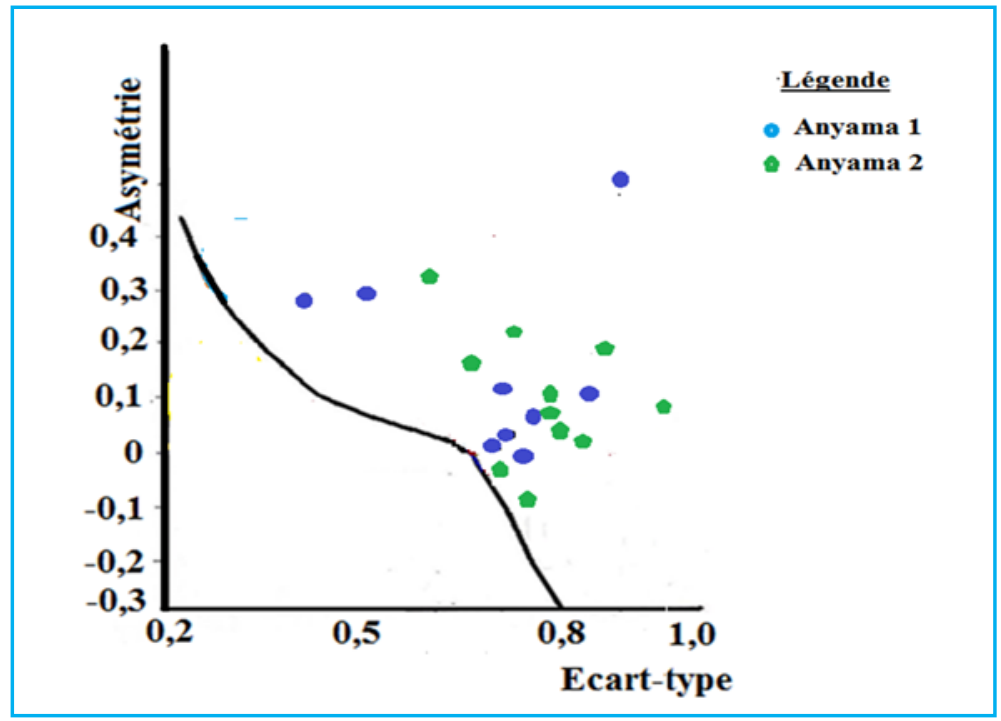

Fiøure 8 : Disbersion des sables d’Anvama sur le diagramme Sk-So de Freidman (1967)

\section{Minéralogie des argiles}

L’examen des caractéristiques minéralogiques générales des niveaux argileux est rendu possible grâce à la diffractométrie des rayons X. Sept échantillons ont été sélectionnés dont ceux des côtes $17 \mathrm{~m}$ et $114 \mathrm{~m}$ pour le puits An1 et ceux des côtes $114 \mathrm{~m}, 110 \mathrm{~m}, 67 \mathrm{~m}, 55 \mathrm{~m}$ et $17 \mathrm{~m}$ pour le puits An2. L'identification des pics est faite à l'aide du logiciel DIFFRACT plus EVA basé sur les fiches PDF (Powder Diffraction Files) anciennement appelées fiches JCPDS (Joint commutee Powder Diffraction Society). Le dépouillement des diffractogrammes a révélé, au niveau du puits An1 (figure 9), la présence de deux minéraux que sont le quartz et la kaolinite. Les plus grands pics sont ceux du quartz qui indiquent leur abondance dans les sédiments. La kaolinite se retrouve cependant associée à un autre minéral argileux qui est l'illite à $114 \mathrm{~m}$. 


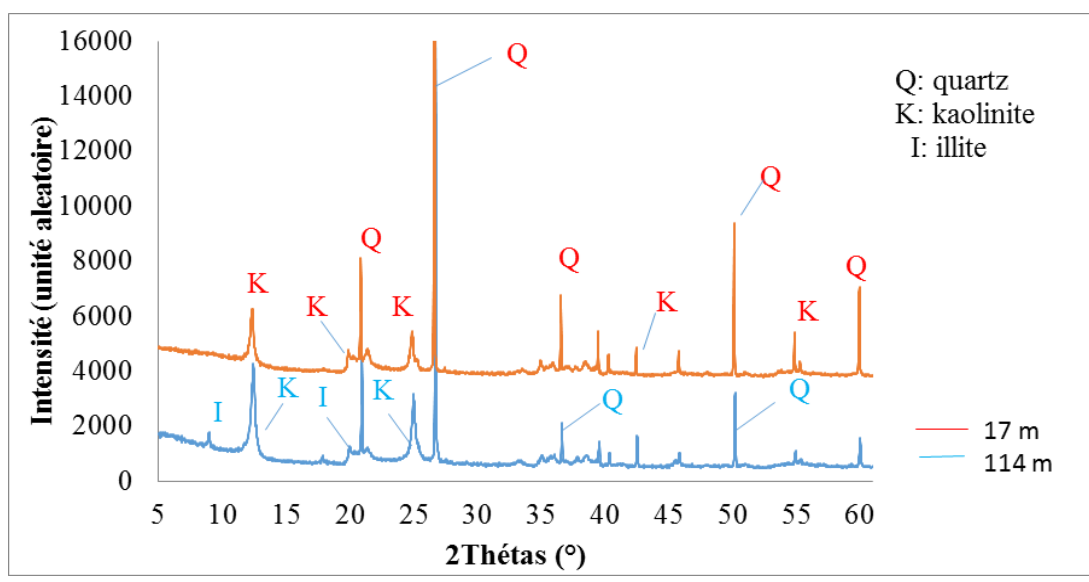

Figure 9 : Diffractogrammes des argiles du puits Anyama 1

Dans le second puits, les diffractogrammes (figure 10) présentent par ordre d'importance, le quartz, la kaolinite, l'illite, le gypse, le rutile ou l'anatase et les sulfures ferrifères de calcium. L'examen détaillé des diffractogrammes indique que :

- le quartz est présent dans tous les échantillons, il est très représentatif. Bien qu'ayant les plus grands pics, Il présente aussi de nombreux pics moyens ;

- la kaolinite est très présente dans les cinq échantillons du profil ;

- l’illite et le gypse sont présents dans les échantillons des côtes 114 $\mathrm{m}$ et $110 \mathrm{~m}$;

- le rutile ou l'anatase et les sulfures : ils présentent de petits pics et sont présent à $114 \mathrm{~m}$.

Les argiles des puits étudiés sont donc composés de quartz et de kaolinite avec des traces de de l'illite, gypse, rutile ou anatase et de sulfate de calcium hydraté.

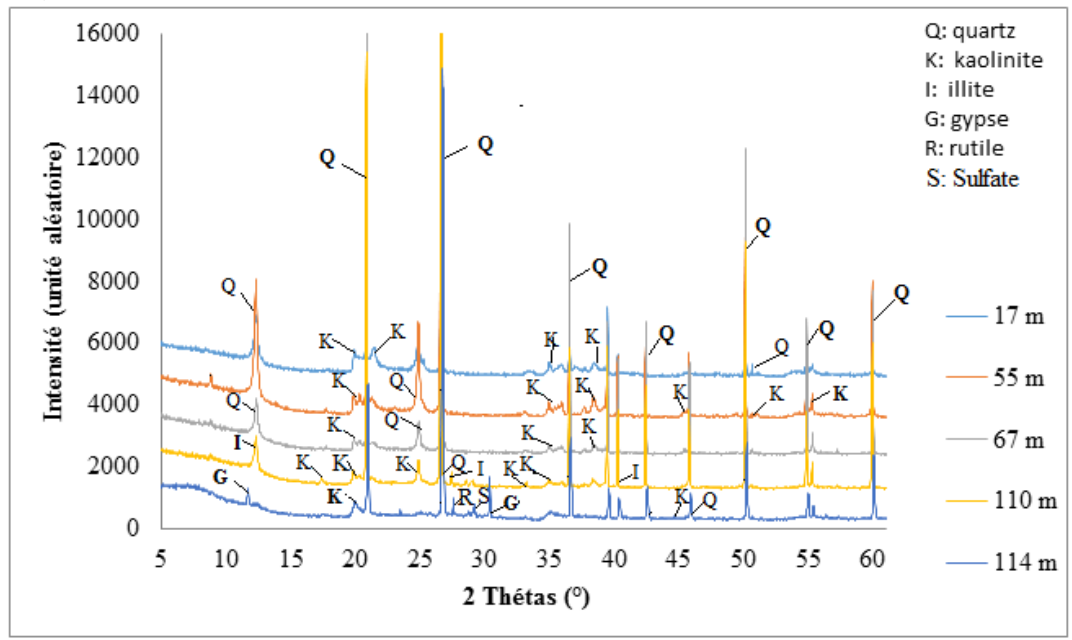

Figure 10 : Diffractogrammes des argiles du puits Anyama 2 


\section{Discussion}

A l'issu de l'analyse lithologique, quatre faciès répondent à des processus de dépôt bien distincts dans la région d'Anyama. Ce sont les argiles (bariolées et grisâtres), les sables quartzeux, les sables argileux et la terre de barre. La corrélation des puits témoigne d'une intercalation de sédiments mixtes (sables argileux et argile grisâtre) entre le niveau de sable quartzeux et l'argile bariolée. Il est donc probable que la couche de sable argileux sur laquelle la terre de barre dans le puits An2 soit la même que l'épaisse couche de sable dans le puits An1. En d'autres termes, la sédimentation s'est faite dans deux chenaux au niveau du puits An2 et termine dans un chenal au niveau de An1. Par ailleurs, la lithologie de la région d'Anyama, à quelque différence près, est semblable à celle décrite à Adjamé, à Yopougon et à Abobo par Gbangbot (2012).

L'alternance de faciès argileux, de faciès sableux moyens à grossiers suggère un hydrodynamisme local très contrasté avec soit, une variation de l'intensité des crues, soit des déplacements latéraux des lits de rivières (Vigreux et al., 2011). Pimmel (1984), va dans le même sens que Vigreux et al. (2011) quand elle affirme que les brusques changements de l'énergie et de la compétence du courant d'écoulement provoquent l'alternance de niveaux sableux et argileux.

Les courbes cumulatives semi-logarithmiques des sables présentent le faciès de type hyperbolique ou sigmoïde. Selon Tricart (1965), le dépôt s'est fait suite à la variation de la compétence du courant de transport. Toujours dans le même sens, Rivière (1977) affirme que le faciès hyperbolique indique une réduction progressive de la force de l'agent de transport. Cet agent qui était initialement capable de transporter les grains d'une certaine dimension ne l'est plus suite à une réduction de sa vitesse, donc de sa force de transport, par conséquent, il abandonne les grains sur place.

Notons aussi que les courbes cumulatives sont à pentes redressées. Ceci témoigne des sables bien classés et un milieu peu agité, tel qu'indiqué par Saidi et al. (2004).

La forme de la courbe de fréquence indique le degré de classement du sédiment. Dans le cas de notre étude, les courbes sont plus ou moins étalées, unimodales, pour la plupart. L'explication qui en découle est que les sables étudiés n'ont pas un bon classement, car, il est dit d'un sable bien classé lorsque la courbe de fréquence est effilée. Ce sont des sables formés d'une seule population comme en témoignent les courbes unimodales.

Les diagrammes de Passega montrent que la majorité des sables est placée dans deux segments à savoir les segments $\mathrm{PO}$ et $\mathrm{ON}$. Le segment PO indique que les sédiments sont transportés par suspension de fond et roulement, alors que le segment ON témoigne des sédiments transportés par 
roulement. En somme, deux modes de transport sont identifiés. Ce sont le transport par suspension de fond et le transport par roulement.

Concernant les environnements de dépôt, les diagrammes de Freidman (1967) indiquent que les sables quartzeux étudiés sont des dépôts de type fluviatile. Ce qui est en adéquation avec les travaux de Assalé (2013) qui stipule que les dépôts sont de type fluviatile au Nord de la faille des lagunes.

Par ailleurs, la minéralogie des argiles a révélé qu’elles sont essentiellement constituées de quartz et de kaolinite. Ce sont donc des argiles kaoliniques silteuses. Un constat est à souligner, c'est que ces argiles sont bariolées en profondeur. C'est la conséquence de l'écoulement irrégulier de l'eau qui a créé un lessivage partiel des sédiments et une distribution aléatoire des oxydes et hydroxydes de fer (Assalé, 2013). Ce sont des dépôts du Plio-pléistocène. Aussi, en amont (puits Anyama 2), l'argile kaolinique bariolée se trouve associée à l'illite, le gypse, le rutile ou l'anatase et des sulfures ferrifères de calcium qui constituent les minéraux associés. En aval, elle est associée qu’à l'illite. Un appauvrissement en éléments associés se fait de l'amont vers l'aval. Le kaolin étant un minéral très stable, se transforme essentiellement dans les altérites par altération des feldspaths alcalins et les micas.

\section{Conclusion :}

La caractérisation des formations de la région d’Anyama a mise en évidence quatre principaux faciès qui sont le faciès argileux, le faciès sableux et le faciès des sédiments mixtes (sables argileux), le tout recouvert de la terre de barre. La région d'Anyama est un paléochenal dans lequel le dépôt s'est effectué avec un brusque changement d'énergie et de la compétence du courant d'écoulement provoquant l'alternance de niveaux sableux et argileux. Concernant les niveaux sableux, la répartition spatiale et granulométrique de ceux-ci indique que les sables d'Anyama sont de taille grossière à rarement moyenne presque symétriques à asymétriques vers les éléments fins, moyennement à assez bien classés. La suspension de fond et le roulement ont été les modes de transport de ces sédiments déposés dans un milieu fluviatile. La minéralogie des argiles a montré que les argiles sont de nature kaolinique et silteuse associées à d'autres minéraux comme l’illite, le gypse, le rutile ou l'anatase et les sulfates de calcium hydratés.

\section{References :}

Assalé F. Y. P., Mondé S., Aka K., Koffi T., Kplohi Y. L. H., Ablé J., Féa I. et Ombléa H. L., 2013. Caractérisation lithostratigraphique des sondages Adia 5 et Adia 6 d’Adiaké : mise en évidence des mouvements eustatiques et 
influence des glauconites sur les propriétés physiques des sables. European Scientific Journal, Vol.9, N.17, pp. 332-349.

Assalé F. Y. P., 2013. Caractérisation sédimentologique, palynologique, géochimique et paléoenvironnementale des formations de l'est du bassin onshore de Côte d'Ivoire. Thèse de Doctorat de l’Université Félix Houphouët Boigny, Abidjan, Côte d’Ivoire, 361 p.

Bamba M. K., Digbehi Z. B., Sombo B. C., Goua T. E. et N’Da L. V., 2011. Foraminifères planctoniques, biostratigraphie et paléoenvironnement des dépôts albo-turonien de la Côte d'Ivoire, Afrique de l'Ouest. Revue de Paléobiologie, Genèse Vol. 30, N¹ pp 1-11.

Bessoles B., 1977. Géologie de l’Afrique : le craton Ouest-africain. Mém. BRGM., n॰88, 402p.

Digbehi Z. B., Affian K. et Yacé P., 1993. Contribution to the sedimentological study of the albo-Cenomanian marine series in Cote d'Ivoire onshore basin. Ann. Nat. Biol. Sci. Abidjan Univ., XXV-B: 25-40.

Digbehi Z. B., Carbonnel G. and Dufaure P., 1994. Contribution to the microfaunistic study (ostracods, foraminifera) to paleogeographic reconstruction of the Côte d'Ivoire “onshore” Basin on Paleocene-Eocene boundary. J. Micropaleontol., 36(3) : 225-240.

Digbehi Z. B., Affian K., Monde S., Pothin K. B. K. et Aka K., 2001. Analyse sédimentologique de quelques faciès du « continental terminal » de la région de Bingerville, environ d'Abidjan, Côte d'Ivoire. Bioterre, Rev. Inter. Sci. de la vie et de la terre, Abidjan (volume 2). $\mathrm{N}^{\circ} 1$. pp. 70-84.

Digbehi Z. B., Ouffouet K. B., Sombo B. C. and Yao N. J-P., 2011. Biostratigraphical Analysis and Palaeoenvironmental Reconstruction of Cenomanian-Turonian Boundary in Côte d'Ivoire Onshore Sedimentary Basin, West Africa. An International Multidisciplinary Journal, Ethiopia, Vol. 5 (6), Serial No. 23, pp. 361-380.

Friedman G. M., 1967. Dynamic processes and statistical parameters compared for size frequency distribution of beach and river sands. J. Sed. Ped., 37, pp. 327-354.

Folk R. \& Ward W., 1957. Brazors river bors, a study in significance of grain size parameters. J.Sedim. Petrol., Vol. 27, pp. 13-27.

Gbangbot J-M. K., Digbehi Z. B., Yao N. J-P., Mondé S. et Yao A. N., 2012. Lithostratigraphie des Dépôts de Subsurface des régions de Bingerville et d'Assinie, Sud et Sud-est, Basse Côte d'Ivoire. Essai de Comparaison des Environnements de Dépôts au Cours du Tertiaire. European Journal of Scientific Research ISSN 1450-216X, Vol. 86, N¹, pp.4152.

Goua T. E., 1997. Biostratigrphie et évolution paléoenvironnementale des séries maastrichtiennes et paléocènes dans le bassin sédimentaire côtier. Thèse d'Etat en géologie, Univ. Bourgogne, centre des sciences de la terre, $354 \mathrm{p}$. 
Martin L. et Tastet J. P., 1972. Le Quaternaire littoral du plateau continental de Côte d'lvoire. Rôle des mouvements tectoniques et eustatiques. Bull. Ass. Sénég. Et. Quatern. Ouest Afr., 33-34 : 17-32.

N’Da L. V., Saint-Marc P., Klasz I. et Goua T., 1995. Données micropaléontologiques sur le passage Crétacé-Tertiaire de Côte d'Ivoire. Rév. Esp. Micropal., vol.XXVII, n³, pp.137152.

Passega R., 1957. Texture as characteristic of clastic deposition. Ann. Assoc. Petrol. Geol.

Pimmel A., 1984. Contribution à l'étude sédimentologique des mangroves de Casamance (Sénégal) : étude granulométrique et exoscopique. Thèse de Doctorat de spécialité ( $3^{\text {ème }}$ cycle) en géologie, Institut de Géographie, Université Louis Pasteur, Strasbourg, France, 132 p.

Rivière A., 1977. Méthodes granulométriques : techniques et interprétation. Ed Masson, Paris, New York, Barcelone, Milan, 149p.

Saaidi E., 1991. Traité de sédimentologie, pétrographie, environnements sédimentaires. Editions Afrique Orient, 399 p.

Saidi H., Brahim M. et Gueddari M., 2004. Caractérisation granulométrique et minéralogique des sédiments de surface de la Frange littorale Sidi Bou Said-la Goulette. Bull Inst. Natn. Scien. Tech. Mer de Salammbô, vol. 31, pp 97-106.

Tagini B., 1971. Esquisse structurale de la Côte d'Ivoire. Essai de géotectonique régionale. Thèse Doc. D’Etat, Lausanne, 302 p.

Tagini B., 1972. Notice explicative à la carte de la Côte d'Ivoire au 1/200000. Rapport interne Soc. Dev. Min. (SODEMI).

Tricart J., 1965. Principes et méthodes de la géomorphologie, Masson et Cie, Paris.22.

Vigreux T., Aoustin D. et Flotté P., 2011. Enregistrement sédimentaire et environnement holocène de la plaine alluviale du Giessen (Scherwiller, BasRhin, Alsace). Quaternaire, 22, (2), 2011, pp. 129-145.

Yacé I., 2002. Initiation à la géologie. L'exemple de la Côte d'Ivoire et de l'Afrique de l'Ouest. Edition CEDA, 183 p.

Yao N. J-P., Digbéhi Z. B., Mondé S., Kra A. C., Aka K., Bléoué N., Tea Y. J., Kplohi Y. L. H. et Duffi K. L., 2011. Etude Sédimentologique et Esquisse Paléoenvironnementale des Formations de Fresco. Sciences \& Nature, Vol. $8, \mathrm{~N}^{\circ} 1: 73-84$. 